Ann. Génét. Sél. anim., I972, 4 (4), 515-521..

\title{
LOCALISATION DES SUBSTITUTIONS D'ACIDES AMINÉS DIFFÉRENCIANT LES VARIANTS A ET B DE LA CASÉINE $x$ BOVINE
}

\author{
F. GROSCLAUDE, Marie-Françoise MAHÉ, J.-C. MERCIER \\ et B. RIBADEAU-DUMAS \\ Laboratoire de Génétique biochimique et Laboratoire de Recherches sur les Protéines, \\ Centre national de Recherches zootechniques, I. N. R. A., \\ 78350 Jouy en Josas
}

\section{RÉSUMÉ}

Comme le suggéraient les résultats de Woychik et al. (I966), DE Koning et al. (1966) et Hill et al. (1970), les caséinomacropeptides des variants A et B de la caséine $x$ bovine diffèrent par deux substitutions d'acides aminés: Ala $(\varkappa \mathrm{B}) \rightarrow \operatorname{Asp}(\varkappa \mathrm{A})$ et Ile $(\varkappa \mathrm{B}) \rightarrow \operatorname{Thr}(\varkappa \mathrm{A})$.

Ces substitutions affectent respectivement la $22^{\mathrm{e}}$ et la $34^{\mathrm{e}}$ position à partir de l'extrémité $\mathrm{COOH}$-terminale du caséinomacropeptide, qui est également celle de la caséine $x$.

D'un point de vue génétique, les deux variants de la caséine $x$ assurent donc le marquage de la partie terminale du locus $x$-Cn.

\section{INTRODUCTION}

Les techniques d'électrophorèse en gel ont permis de mettre en évidence un polymorphisme génétique au niveau de chacune des trois espèces principales de caséine bovine, les caséines $\alpha_{s_{1}}$, $\beta$ et $\chi$ (voir les revues bibliographiques d'AschaFFENBURG, I968 et de THompson, I970). Les quatre variants génétiques de caséine $\alpha_{\boldsymbol{B}_{1}}$, les cinq variants de caséine $\beta$ et les deux variants de caséine $x$ détectés à ce jour chez Bos taurus sont déterminés par les allèles des trois loci de structure, $\alpha_{\delta_{1}}-\mathrm{Cn}, \beta$-Cn et $x$-Cn. Ces loci présentent la particularité d'être très étroitement liés, au point que leurs allèles ségrègent comme s'ils formaient une seule unité génétique (GroscLAUDE et al., I964, I965; KING et al., I965 ; LARSEN et Thymann, I966).

Nous avons suggéré, dans des publications antérieures (GROSCLAUDE et al., I966, r969) que la localisation des mutations correspondant à tous ces variants pouvait contribuer à préciser la disposition relative des trois loci de structure considérés. 
Pour localiser ces mutations, il suffit, en vertu du principe de colinéarité entre un gène de structure et la protéine correspondante, de localiser, dans chacune des chaînes polypeptidiques, les altérations propres aux différents variants génétiques.

Nous venons de caractériser de la sorte tous les variants des caséines $\alpha_{s_{1}}$ et $\beta$ connus à ce jour (GRosclaUde et al., I972).

En ce qui concerne la caséine $x$, les compositions en acides aminés établies par Woychik et al. (Ig66) montrent que, par rapport à celle du variant $\varkappa \mathrm{B}$, la chaîne polypeptidique du variant $x \mathrm{~A}$ comporte d'une part un résidu alanyle et un résidu isoleucyle en moins, et d'autre part un résidu thréonyle et un résidu aspartyle ou asparaginyle en plus; Woychik et al. ont suggéré que les deux variants différaient par les substitutions Ala $\rightarrow$ Asp et Ile $\rightarrow$ Thr, car, dans cette hypothèse, chacune des mutations correspondantes peut n'impliquer que la substitution d'une seule paire de nucléotides. DE KonING et al. (I966) ont montré par ailleurs que ces substitutions affectaient le caséinomacropeptide, c'est-à-dire la partie COOH-terminale de la caséine $x$, ce qui a été confirmé par HIL et al. (I970). Le présent travail qui se réfère à la séquence du caséinomacropeptide du variant $x \mathrm{~B}$ établie par MERCIER et $a l$. (I972), précise la localisation des deux substitutions d'acides aminés qui différencient les variants $x \mathrm{~A}$ et $x \mathrm{~B}$.

\section{MATÉRIEL, ET MÉTHODES}

La caséine $x \mathrm{~A}$ a été préparée suivant Zittle et Custer (1963) à partir du lait d'une vache de race Française Frisonne Pie-Noire, homozygote pour le complexe génique

$$
\alpha_{s_{1}}-\mathrm{Cn}^{\mathrm{B}}-\beta-\mathrm{Cn}^{\mathrm{A}^{2}}-x-\mathrm{Cn}^{\mathrm{A}}
$$

La fraction dépourvue de glucides, $\chi \mathrm{A}_{1}$, a été obtenue par chromatographie de la caséine $\chi \mathrm{A}$ sur DEAE-cellulose en milieu urée et $\beta$-mercaptoéthanol (MERcier et al., 1968). Son homogénéité a été vérifiée par électrophorèse en gel d'amidon selon Schmidt (1964).

Les autres techniques utilisées dans ce travail ont toutes été décrites dans nos publications antérieures : électrophorèse et chromatographie sur papier, chromatographie sur colonne de résine Dowex, dosage des acides aminés, dosage du phosphore : Grosclaude et al. (1970); hydrolyse par la leucine aminopeptidase, par la carboxypeptidase A et par la pepsine, dégradation soustractive d'Edman : MERCier et al. (1970 a) ; hydrolyse par le bromure de cyanogène : MERCIER et al. (r97o $b$ ) ; hydrolyse acide ménagée, utilisation de la phosphatase alcaline : Mercier et al. (r97I) ; hydrolyse par la thermolysine: Ribadeau-Dumas et al. (r97I) ; utilisation de l'aminopeptidase M : Ribadeau-Dumas et al. (1972).

L'origine des enzymes et produits utilisés a été donnée par MERCIER et al. (1972).

La nomenclature des peptides résultant de l'hydrolyse d'un peptide plus long fait intervenir, dans l'ordre, trois éléments : a) la dénomination du peptide de départ, $b$ ) les initiales du nom de l'enzyme ou du réactif ayant servi à l'hydrolyse $(\mathrm{Th}=$ thermolysine ; $\mathrm{CN}=$ bromure de cyanogène), c) un numéro d'ordre, attribué en fonction du résultat de l'opération de fractionnement effectuée. Le numérotage des peptides se fait dans l'ordre d'élution pour une chromatographie préparative sur colonne, de l'anode vers la cathode pour une électrophorèse préparative sur papier, et dans le sens des mobilités croissantes pour une chromatographie préparative sur papier. Par ailleurs l'appellation d'un peptide comporte, si besoin est, le nom du variant génétique ayant servi à le préparer.

\section{RÉSULTATS}

Comme pour l'étude de la séquence du caséinomacropeptide de la caséine $x \mathrm{~B}$ (MERCIER et al., I972), nous n'avons pas opéré sur le caséinomacropeptide lui-même mais sur le peptide $x A_{1} \mathrm{CN}_{2}$, l'un des 3 peptides résultant de 1'hydrolyse de la ca- 


\section{Peptide $\times A_{1} C N 2$}

Composition en acides aminés

Composition de $\varkappa \mathrm{B}_{1} \mathrm{CN} 2$ peptide homologue de $x B_{1}$ (MERCIER et al., 1972)

\section{Peptide $x A_{1}$ CN2Th1}

Composition en acides aminés

Composition de $\chi \mathrm{B}_{1} \mathrm{CN} 2 \mathrm{Th} 1$ peptide homologue de $x \mathrm{~B}_{1}$ (MERCIER et al., 1972)

\section{Peptide $x A_{1} C N 2 T h 1 \mathrm{a}$}

Composition en acides aminés

Séquence

Edman après déphosphorylation Stade 1

Stade 2

Stade 3

Stade 4

Stade 5

Peptide $\varkappa A_{1} C N 2 T h_{1} \mathrm{~b}$

Composition en acides aminés

\section{Peptide $\varkappa A_{1}$ CN2Th2}

Composition en acides aminés

Composition de la séquence homologue de $\chi \mathrm{B}_{1}$ (Mercier et al., 1972)

\section{Peptide $x A_{1} C N 2 T h 2 P 1$}

Composition en acides aminés

Carboxypeptidase $\mathrm{A}^{* *}$

Peptide $\mathrm{A}_{1} \mathrm{CN} 2 \mathrm{Th} 3$

Composition en acides aminés

Composition de la séquence homo- $T h r_{3}, \mathrm{Ser}_{2}, \mathrm{Glx}_{2}, \mathrm{Pro}_{2}, \mathrm{Gly}, \mathrm{Ala}_{2}, I l e_{2}$. logue de $\chi_{1} \mathrm{~B}_{1}$ (MERcier et al., 1972)

Leucine aminopeptidase

Carboxypeptidase $\mathrm{A}^{* *}$
Asx, 5,01(5) ; Thr, 11,50(12) ; Ser, 5,77(6) ; Glx, 10,21(10) ; Pro, 7,64(8) ; Gly, 1,03(1); Ala, 4,85(5) ; Val, 5,95(6) ; Ile, 5,61(6) ; Leu, 1,04(1) ; Lys, $2,80(3) ; \mathrm{P}, 0,97(1)$.

Asx, 3,93(4) ; Thr, 10,62(11) ; Ser, 5,75(6) ; Glx, 10,04(10) ; Pro, 8,16(8) ; Gly, 1,07(1);Ala, 6,03(6); Val, 5,83(6);Ile, 6,60(7); Leu, 0,97(1); Lys, $2,87(3) ; \mathrm{P}(1)$.

Asx, 1,75(2) ; Thr, 1,09(1) ; Ser, 1,61(2) ; Glx, 4,06(4) ; Pro, 3,03(3) ; Val, 1,00(1); Ile, 1,52(2); Leu, 1,00(1); $\mathrm{P}^{*}$.

Asx, $0,96(1)$; Thr, 1,01(1) ; Ser, 1,80(2); Glx, 4,02(4) ; Pro, 2,94(3) ; Ala, $1,00(1)$; Val, $0,73(1)$; Ile, 1,62(2); Leu, 1,01(1).

Asx, 1,00(1) ; Ser, 0,95(1); Glx, 2,08(2) ; Pro, 0,86(1) ; Leu, 0,77(1); $\mathrm{P}^{*}$.

Leu - Glu - Asp - SerP - Pro - Glu

Asx, 0,89(1) ; Ser, 1,00(1); Glx, 2,10(2) ; Pro, 1,28(1); Leu, 0,23(0).

Asx, 0,84(1) ; Ser, 1,00(1); Glx, 1,55(1) ; Pro, 1,26(1).

Asx, $0,37(0)$; Ser, 1,00(1) ; Glx, 1,38(1) ; Pro, 1,12(1).

Asx, 0,28(0); Ser, 0,42(0); Glx, 1,30(1) ; Pro, 0,97(1).

Asx, 0,27(0) ; Ser, 0,40(0); Glx, 1,30(1) ; Pro, 0, 70(0-1).

Asx, 0,94(1) ; Thr, 0,94(1) ; Ser, 1,09(1) ; Glx, 2,29(2) ; Pro, 2,29(2); Val, $0,90(1)$; Ile, 1,52(2).

Thr, 4, 46 (5) ; Ser, 2, 58 (3) ; Glx, 3, 02 (3) ; Pro, 2, 02 (2) ; Gly, 0, 91 (1); Ala, 1, $87(2) ; \mathrm{Val}, 1,00(1) ;$ Ile, 1, 07 (1).

$T h r_{4}, \mathrm{Ser}_{3}, \mathrm{Glx}_{3}, \mathrm{Pro}_{2}, \mathrm{Gly}, \mathrm{Ala}_{2}, \mathrm{Val}, \mathrm{Ile}_{2}$.

Thr, 3, 29 (4) ; Ser, 1, 58 (2) ; Glx, 1, 85 (2) ; Pro, 1, 85 (2) ; Gly, 0, 99 (1); Ala, 1, 03 (1); Ile, 0, 76 (1).

( $5 \mu 1 ; 1$ h) Glu, 0, 32 ; Thr, 0, 19.

Thr, 4, $12(4)$; Ser, 2, 01 (2) ; Glx, 2, 35 (2) ; Pro, 1, 87 (2) ; Gly, 0, 90 (1) ; Ala, 1, $66(2) ;$ Ile, 1, $00(1)$.

$(2 \mu l ; 15 \mathrm{~h})$ Ile, 0,$80 ;$ Ala, 0,54 .

$(2 \mu l, 15$ h) Thr, 0, 45 ; Glu, 0, 28 ; Ala, $0,28$.

* Mis en évidence par électrophorèse sur papier, sous la forme de phosphosérine, après hydrolyse acide ménagée.

** $\mu l$ de solution commerciale/0,1 $\mu$ mole de peptide. 
séine $\varkappa \mathrm{A}_{1}$ par le bromure de cyanogène et qui correspond au caséinomacropeptide amputé de son résidu méthionyle $\mathrm{NH}_{2}$-terminal. Ce peptide a été préparé comme son homologue $x \mathrm{~B}_{1} \mathrm{CN} 2$ (MERCIER et al., I972). La confrontation de la composition en acides aminés de $x \mathrm{~A}_{1} \mathrm{CN} 2$ avec celle de $x \mathrm{~B}_{1} \mathrm{CN} 2$ (tabl. I) confirme les conclusions de De Koning et al. (I966) et HrLI et al. (I970) : par rapport à $x \mathrm{~B}_{1} \mathrm{CN} 2, x \mathrm{~A}_{1} \mathrm{CN} 2$ possède un résidu alanyle et un résidu isoleucyle en moins, un résidu thréonyle et un résidu aspartyle ou asparaginyle en plus.

Pour localiser ces substitutions, le peptide $x \mathrm{~A}_{1} \mathrm{CN} 2$ a été hydrolysé par la thermolysine (II $\mu$ Moles; E/S : I $/ 200 ; 40^{\circ} \mathrm{C}$; I heure) et 1'hydrolysat ainsi obtenu chromatographié sur colonne de Dowex $50 \mathrm{WX} 2$ (MERCIER et al., I972). Les principaux résultats de cette chromatographie figurent sur le tableau $I$ : la première fraction du chromatogramme nous a fourni le peptide $x_{1} \mathrm{ANN}_{2} \mathrm{Th} \mathrm{I}$ dont la composition en acides aminés est, à une substitution $\mathrm{Ala} / \mathrm{Asx}$ près, identique à celle du peptide $\varkappa_{1} \mathrm{~B}_{1} \mathrm{~N} 2 \mathrm{ThI}$. La seconde fraction nous a fourni le peptide $x \mathrm{~A}_{1} \mathrm{CN} 2 \mathrm{Th} 2$ dont la composition, obtenue après repurification par électrophorèse sur papier, correspond, à une substitution Ile/Thr près, à la somme des compositions des peptides $x \mathrm{~B}_{1} \mathrm{CN} 2 \mathrm{Th} 2$ et $x \mathrm{~B}_{1} \mathrm{CN} 2 \mathrm{Th} 3$. La troisième fraction nous a fourni le peptide $x \mathrm{~A}_{1} \mathrm{CN}_{2} \mathrm{Th} 3$ dont la composition correspond, à une substitution Ile/Thr près, à la somme des compositions des peptides $x \mathrm{~B}_{1} \mathrm{CN} 2$ 'Th2 et $x \mathrm{~B}_{1} \mathrm{CN} 2$ Th8. La figure I montre 1'emplacement de ces trois peptides

paracaséine

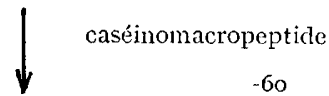

$-70$

$-60$

... -Met-Ala-Arg-His-Pro-His-Pro-His-Leu-Ser-Phe-Met-Ila-Ile-Pro-I'ro-Iys-Iys-Isn-(jlı

$-50$

$-40$

-Asp-Iys-Thr-Glu-Ile-Pro-Thr-Ile-Asn-Thr-Ile-Ala-Ser-Gly-Gilu-Pro-Thr-Ser-Thr-I'ro
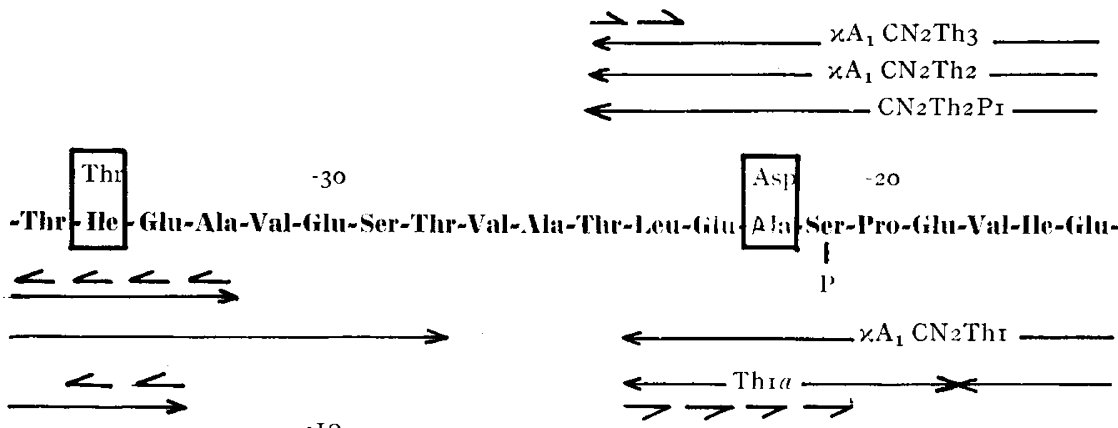

$\cdot$ Io

-Ser-Pro-Pro-Glu-Ile-Asn-Thr-Val-Gln-Val-Thr-Ser-Thr-. la-Val. $O H$

ThI $b \longrightarrow$

FIG. I. - Localisation dans le caséinomacropeptide des substitutions d'acides aminés différenciant les variants génétiques de la caséine $\varkappa$ bovine ( $x \mathrm{~A}$ et $\varkappa \mathrm{B}$ ).

La séquence de référence est celle du variant $\chi \mathrm{B}_{1}$ (Mercier et al., 1972)

Les résidus sont numérotés négativement à partir de l'extrémité COOH-terminale du caséinomacropeptide, qui est aussi celle de la caséine $\varkappa$. La séquence du peptide $\varkappa \mathrm{A}_{1} \mathrm{CN}_{2}$ est imprimée en caractères gras. Les deux substitutions mises en évidence et localisées sont encadrées.

$\longleftrightarrow$, peptides ayant servi à localiser ces substitutions; $\longrightarrow$, acide aminé libéré par la leucine aminopeptidase ; $\longrightarrow$, acide aminé libéré par la carboxypeptidase $\mathrm{A} ; \longrightarrow$, acide aminé identifié par la technique soustractive d'Edman; $\downarrow$ liaison dont l'hydrolyse par la présure libère le caséinomacro. peptide. 
dans la séquence du peptide $\mathrm{CN}_{2}$ (ou du caséinomacropeptide). A noter que les autres fractions du chromatogramme nous ont fourni, avec des compositions identiques à celles de leurs homologues $d u$ variant $x B_{1}$, les peptides correspondant au reste de la séquence de $x_{1} \mathrm{AN}_{2}$.

D'autre part, une répétition de cette manipulation effectuée dans des conditions similaires à partir de $7 \mu$ moles de peptide $x \mathrm{~A}_{1} \mathrm{CN}_{2}$, nous a permis d'isoler, à partir d'une fraction éluée juste après celle contenant $x \mathrm{~A}_{1} \mathrm{CN} 2 \mathrm{ThI}$, les peptides $x \mathrm{~A}_{1} \mathrm{CN} 2 \mathrm{Th} I a$ et $x \mathrm{~A}_{1} \mathrm{CN} 2 \mathrm{Th} \mathrm{rb}$, purifiés par chromatographie sur papier, lesquels ne peuvent provenir que de l'hydrolyse partielle du peptide $x \mathrm{~A}_{1} \mathrm{CN} 2 \mathrm{ThI}$ (tabl. I et fig. I).

L'ensemble de ces résultats suggérait que $x \mathrm{~B}_{1} \mathrm{CN}_{2}$ et $x \mathrm{~A}_{1} \mathrm{CN}_{2}$ différaient d'une part par une substitution Ile $\rightarrow$ Thr affectant 1'élément de séquence représenté par le peptide $x \mathrm{~A}_{1} \mathrm{CN} 2 \mathrm{Th} 3$ et d'autre part par une substitution Ala $\rightarrow$ Asx affectant l'élément de séquence représenté par le peptide $x \mathrm{~A}_{1} \mathrm{CN} 2$ ThIa. L'identification exacte et la localisation de ces substitutions sont prouvées par les résultats suivants :

\section{A. - Peptides $x A_{1} C N 2 T h 3$ et $x A_{1} C N 2 T h 2 P 1$}

L'action de la leucine aminopeptidase montre que le seul résidu isoleucyle que contient le peptide $x \mathrm{~A}_{1} \mathrm{CN} 2 \mathrm{Th} 3$ est situé en position $\mathrm{NH}_{2}$-terminale ; par contre, 1'action de la carboxypeptidase $\mathrm{A}$ ne donne pas de résultats concluants.

Le peptide $x \mathrm{~A}_{1} \mathrm{CN} 2$ Th2 2 alors été hydrolysé par la pepsine $(3 \mu$ moles ; E/S : I/50, température ambiante; 24 heures), et 1'hydrolysat ainsi obtenu chromatographié sur colonne de Sephadex, en milieu acide acétique 30 vol. p. Ioo. La première fraction $\mathrm{du}$ chromatogramme nous a fourni le peptide $x \mathrm{~A}_{1} \mathrm{CN} 2 \mathrm{Th} 2 \mathrm{PI}$, qui correspond au peptide $x \mathrm{~A}_{1} \mathrm{CN}_{2} \mathrm{~T}_{3}$ amputé d'un résidu alanyle, résidu qui est, on le voit, en position $\mathrm{COOH}$-terminale dans ce dernier peptide. L'action de la carboxypeptidase A sur le peptide $x \mathrm{~A}_{1} \mathrm{CN}_{2}$ Th2 $\mathrm{PI}$ montre que la séquence $\mathrm{COOH}$-terminale de ce dernier est Thr-Glu $\mathrm{OH}$. Autrement dit, la $34^{\mathrm{e}}$ position à partir de l'extrémité COOHterminale du caséinomacropeptide (tabl. I) est occupée, dans le variant A, par un résidu thréonyle, et non plus, comme dans le variant $B$, par un résidu isoleucyle.

\section{B. - Peptide $x A_{1} C N 2 T h 1 a$}

Les résultats des dégradations soustractives d'Edman montrent que le résidu qui précède le résidu phosphoséryle est un résidu aspartyle ou asparaginyle alors que dans le variant $x \mathrm{~B}_{1}$, la position correspondante $\left(22^{\mathrm{e}}\right.$ à partir de l'extrémité $\mathrm{COOH}$ terminale) est occupée par un résidu alanyle. Nous avons vérifié par électrophorèse sur papier que l'action de 1'aminopeptidase M libérait de l'acide aspartique, et non de 1'asparagine.

\section{CONCLUSION ET DISCUSSION}

Le variant $A$ de la caséine $x$ bovine diffère donc du variant $B$ par deux substitutions d'acides aminés, une substitution Ala $\rightarrow$ Asp et une substitution Ile $\rightarrow \mathrm{Thr}$, ce qui confirme et précise les résultats de WoychIK et al. (I966), DE KONING et al. (I966) et HrL et al. (I970). Ces substitutions affectent respectivement la $22^{\mathrm{e}}$ et $1 \mathrm{a} 34^{\mathrm{e}}$ posi- 
tion de la chaîne polypeptidique de la caséine $x$ à partir de son extrémité $\mathrm{COOH}-$ terminale. Chacune d'elles peut, selon le code génétique, ne dériver que de la mutation d'une seule paire de nucléotides $\left(\mathrm{GC}_{\mathrm{U}}^{\mathrm{C}} \rightarrow \mathrm{GA}_{U}^{\mathrm{C}}\right.$ pour Ala $\rightarrow \mathrm{Asp} ; \mathrm{AU}_{\mathrm{U}}^{\mathrm{C}} \rightarrow \mathrm{AC}_{\mathrm{U}}^{\mathrm{C}}$ pour Ile $\rightarrow$ Thr). La substitution Ile $\rightarrow$ Thr mettant en jeu des résidus neutres, c'est la seule substitution Ala $\rightarrow$ Asp qui est responsable de la différence de mobilité électrophorétique des deux variants $\mathrm{A}$ et $\mathrm{B}$ à $\mathrm{pH}$ alcalin. C'est donc elle, en fait, qui assure le marquage du locus $x$-Cn et, plus précisément, de l'extrémité terminale de ce locus.

Reçu pour publication en mai 1972.

\section{SUMMARY}

\section{LOCAIIZATION OF AMINO-ACID SUBSTITUTIONS DIFFERENTIATING}

\section{THE A AND B VARIANTS OF $x$-CASEIN IN CATTLE}

As was suggested by the results of WoychiK et al. (1966), De Koning et al. (I966) and HrLL et al. (1970) the caseinomacropeptides from the A and B variants of bovine $x$-casein differ by two amino-acid substitutions: Ala $(\varkappa \mathrm{B}) \rightarrow \operatorname{Asp}(\varkappa \mathrm{A})$ and Ile $(\varkappa \mathrm{B}) \rightarrow \operatorname{Thr}(\varkappa \mathrm{A})$.

These substitutions affect the 22 nd and 34 nd positions, respectively, from the COOHterminus of the caseinomacropeptide, which is also the COOH-terminus of $x$-casein.

From a genetical point of view, the two variants of $x$-casein ensure the marking of the terminal part of $x$-casein locus.

\section{RÉFÉRENCES BIBLIOGRAPHIQUES}

Aschaffenburg R., I968. Genetic variants of milk proteins; their breed distribution. J. Dairy Res., 35, 447-46o.

De Koning P. J., VAn Rooijen P. J., Kok A., 1966. Location of amino acid differences in the Genetic Variants of $x$-Casein A and B. Biochem. Res. Commun., 24, 6I6-621.

Grosclaude F., Garnier J., Ribadeau-Dumas B., Jeunet R., I964. Étroite dépendance des loci contrôlant le polymorphisme des caséines $\alpha_{s}$ et $\beta$. C. R. Hebd. Séances Acad. Sci. (Paris), 259, I569I57I.

Grosclaude F., Mahé M.-F., Mercier J.-C., Ribadeau-Dumas B., i972. Caractérisation des variants génétiques des caséines $\alpha_{s_{1}}$ et $\beta$ bovines. Eur. J. Biochem., 26, 328-337.

Grosclaude F., Mercier J.-C., Ribadeau-Dumas B., 1969. Sur la localisation, dans la séquence COOH-terminale de la caséine $\alpha_{s_{1}}$ bovine, de la substitution GLU/GLY différenciant les variants génétiques B et C. C. R. Hebd. Séances Acad. Sci. (Paris), 268, 3I33-3I36.

Grosclaude F., Mercier J.-C., Ribadeau-Dumas B., i97o. Structure primaire de la caséine $\boldsymbol{\alpha}_{s_{1}}$ bovine. Localisation des peptides trypsiques dans les fragments obtenus par hydrolyse trypsique de la caséine maléylée. Eur. J. Biochem., 14, 98-107.

Grosclaude F., Pujolle J., Garnier J., Ribadeau-Dumas B., i965. Déterminisme génétique des caséines $\chi$ du lait de vache ; étroite liaison du locus $x$-Cn avec les loci $\alpha_{s}-\mathrm{C} n$ et $\beta-\mathrm{C} n$. C.R. Hebd. Séances Acad. Sci. Paris, 261, 5229-5232.

Grosclaude F., Pujolle J., Ribadeau-Dumas B., Garnier J., ig66. Analyse génétique du groupe de loci de structure synthétisant les caséines bovines : in : Polymorphismes biochimiques des animaux, 4I5-420. Institut National de la Recherche Agronomique, Paris.

Hill R. J., Naughton M. A., Wake R. G., 197o. The major genetic variant macropeptides of $x$-casein. A comparison of their amino-acid contents and tryptic peptides. Biochem. Biophys. Acta, $200,267-274$.

King J. W. B., Aschaffenburg R., Kiddy C. A., Thompson M. P., I965. Non-independent occurrence of $\alpha_{s_{1}}$ and $\beta$-casein variants of cow's milk. Nature, 206, 324-325.

Larsen B., Thymann M., I966. Studies on milk protein polymorphism in Danish cattle and the interaction of the controlling genes. Acta vet. Scand., 7 , $189-205$. 
Mercier J.-C., Grosclaude F., Ribadeau-Dumas B., i97o a. Structure primaire de la caséine $\alpha_{s_{1}}$ bovine. Séquence de 48 résidus d'acides aminés de l'extrémité COOH-terminale. Eur. J. Biochem., 14, IO8-II9.

Mercier J.-C., Grosclaude F., Ribadeau-Dumas B., r97o $b$. Structure primaire de la caséine $\alpha_{s 1}$ bovine. Enchaînement des peptides obtenus par action du bromure de cyanogène et des peptides résultant de l'hydrolyse de la caséine $\alpha_{s_{1}}$ maléylée. Eur. J. Biochem., 16, 439-446.

Mercier J.-C., Grosclaude F., Ribadeau-Dumas B., r971. Structure primaire de la caséine $\alpha_{s 1}$ bovine. Séquence complète. Eur. J. Biochem., 23, 4I-5I.

Mercier J.-C., Maubois J. L., Poznanski S., Ribadeau-Dumas B., ig68. Fractionnement préparatif des caséines de vache et de brebis par chromatographie sur DEAE cellulose, en milieu urée et 2-mercaptoéthanol. Bull. Soc. Chim. Biol., 50, $521 \cdot 530$.

Mercier J.-C., Uro J., Ribadeau-Dumas B., Grosclavde F., I972. Structure primaire du caséinomacropeptide de la caséine $\varkappa \mathrm{B}_{1}$ bovine. Eur. J. Biochem., 27, 535-547.

Ribadeau-Dumas B., Brignon G., Grosclaude F., Mercier J.-C., I972. Structure primaire de la caséine $\beta$ bovine. Séquence complète. Eur. J. Biochem., 25, 505-5 44.

Ribadeau-Dumas B., Grosclaude F., Mercier J.-C., I97i. Structure primaire de la caséine $\beta$ bovine. Enchaînement des peptides trypsiques et des peptides obtenus par action du bromure de cyanogène. Eur. J. Biochem., 18, 252-257.

Schmidt D. G., r964. Starch-gel electrophoresis of $x$-casein. Biochim. Biophys. Acta., 90, 4II-4I4. Thомpson M. P., I97o. Phenotyping milk proteins : a review. J. Dairy Sci., 53, I34I-I348.

Woychik J. H., Kalan E. B., Noelken M. E., I966. Chromatographic isolation and partial characterisation of reduced $x$-casein components. Biochemistry, 5, 2276-2282.

Zittle C. A., Custer J. H., I963. Purification and some of the properties of $\alpha_{s}$-casein and $x$-casein. J. Dairy Sci., 46, I $183^{-1}$ I 88. 